\section{Vitreous surgery in the management of chronic endogenous posterior uveitis}

RA Scott ${ }^{1}$, RJ Haynes², GM Orr², R J Cooling ${ }^{1}$, CE Pavésio ${ }^{1}$ and DG Charteris

\begin{abstract}
Objectives There is evidence that pars plana vitrectomy (PPV) has a beneficial effect on the clinical course of chronic endogenous posterior uveitis (EPU) possibly by physically removing any resident inflammatory cells with the vitreous. We assessed the anatomical and therapeutic effects of PPV performed on patients with chronic EPU for any indication. Patients and methods Retrospective review of 41 eyes of 38 consecutive patients with EPU who underwent a PPV for any reason, over a 5year period. The mean age of the patients was 36.2 years, $46 \%$ of the eyes had intermediate uveitis, $32 \%$ panuveitis, and $22 \%$ posterior uveitis. The visual acuity, disease activity, and the requirement for medications to control it were recorded for 12 months pre- and postoperatively.
\end{abstract}

Results Overall, $61 \%$ of the eyes gained more than 2 Snellen lines $(P<0.001)$ and the incidence of cystoid macular oedema (CMO) significantly reduced from 44 to $20 \%(P<0.05)$. Postoperatively, there was a significant decrease in the recurrence rate of intermediate uveitis, posterior uveitis, and panuveitis $(P<0.001)$. The use of systemic and local depot immunosuppressive agents did not change over the study period, although the use of topical agents increased $(P<0.05)$.

Conclusion PPV appears to have a beneficial effect on the clinical course of EPU in selected cases. This may be mediated by the physical clearance of inflammatory debris, the anti-inflammatory effect of replacing vitreous by aqueous humour, by a reduction of CMO and/or the anatomical correction of sight-threatening retinal pathology.

Eye (2003) 17, 221-227. doi:10.1038/

sj.eye. 6700299

Keywords: vitrectomy; uveitis; cystoid macular oedema

\section{Introduction}

Endogenous posterior uveoretinitis (EPU) encompasses the autoimmune inflammatory syndromes of the posterior segment of the eye associated with vitritis, choroiditis, retinal vasculitis, cystoid macular oedema, and optic nerve head oedema. EPU is characterised by an exaggerated immune response leading to tissue destruction, which may be localised to the eye or comprise part of a systemic disease. ${ }^{1,2}$

EPU responds to general inhibition of the immune response with systemic steroids as well as inhibitors of T-cell activation such as cyclosporine A or FK506. The population of autoreactive lymphocytes lodged in the vitreous in chronic uveitis appears to be unable to exit the eye and does not readily undergo apoptosis. ${ }^{3,4}$ It has been proposed that this induces the collection of a 'sump' of inflammatory cells lodged in the vitreous that may be reactivated to cause an uveitic exacerbation. ${ }^{5}$

Pars plana vitrectomy (PPV) physically removes the vitreous humour and in the case of EPU, any inflammatory cells that might be trapped in it. There is clinical evidence that PPV has a beneficial effect on the clinical course of chronic uveitis. There is a reported improvement in visual acuity, 6,7 with a reduction in the incidence of cystoid macular oedema. 8 ,9 The number of uveitic exacerbations is also reduced with fewer immunosuppressive medications required. ${ }^{10-13}$ PPV in uveitis can also be a useful aid in diagnosis by providing a view of the fundus and biopsy material for laboratory analysis. This can reveal a diagnosis in approximately $30-50 \%$ of cases where the fundus cannot be otherwise visualised. ${ }^{14}$

Potential complications of PPV in uveitis include postoperative uveitic exacerbations, retinal detachment, and the induction and progression of lens opacities. Despite these risks, PPV is indicated as part of the surgical management of certain sequelae of EPU,
${ }^{1}$ Moorfields Eye Hospital London EC1V 2PD, UK

${ }^{2}$ Queens Medical Centre Nottingham NG7 2UH, UK

Correspondence:

DG Charteris

Moorfields Eye Hospital London EC1V 2PD, UK

Tel/Fax: + 44 (0) 207566

2285

E-mail: david.charteris@ moorfields.nhs.uk

Received: 12 February 2002 Accepted: 13 May 2002 
including persistent vitreous inflammatory debris, vitreous haemorrhage, traction retinal detachments, and epiretinal membranes. ${ }^{15}$ Our aim was to assess the anatomical and therapeutic effects of PPV for patients with chronic EPU who required vitreous surgery for any indication.

\section{Patients and methods}

This was a retrospective review of 41 eyes (19 right, 22 left) of 38 consecutive patients with EPU who underwent PPV for any indication at Moorfields Eye Hospital, London and Queens Medical Centre, Nottingham between January 1992 and December 1997. The mean age of the patients was 36.2 years (range 9-68), 20 were males and 18 females. Six eyes had undergone a previous cataract extraction, five with a posterior chamber intraocular lens implant (IOL) and one was aphakic. Two eyes had a history of glaucoma; at the time of operation one was medically controlled and the other had undergone a trabeculectomy but also required topical medication.

The primary indication for the PPV was for media opacities (including visually significant floaters) in 18 eyes, retinal detachment in eight eyes, epiretinal membrane in nine eyes, and vitreous haemorrhage in six eyes. The uveitic syndromes included 19 eyes (46\%) with intermediate uveitis, 13 eyes (32\%) with panuveitis, and nine $(22 \%)$ with posterior uveitis. Of the nine eyes with posterior uveitis, seven were idiopathic, one associated with multiple sclerosis, and one with systemic sarcoidosis.

Patients were examined preoperatively to confirm the clinical diagnosis. At each visit the best-corrected Snellen visual acuity, the results of slit-lamp biomicroscopy with fundal examination and applanation tonometry were recorded. Cystoid macular oedema was assessed both clinically and by fluorescein angiography; since the timing of angiography was not standardised, the clinical findings were used for analysis. The vitreous was graded using Nussenblatt's classification from 0 to $4+$ according to the fundal structures visible on indirect ophthalmoscopy through a dilated pupil with a 20dioptre lens. ${ }^{16}$ For statistical analysis, visual acuity was graded from 1 to 10 corresponding to the Snellen visual acuity, ranging from $6 / 6$ or better to no light perception. The primary outcome of a significant change in vision was designated as an improvement or loss of more than 2 Snellen lines.

The percentage of eyes that suffered an uveitic exacerbation per month, and the number and type of immunosuppressive agents taken by each patient per month were recorded over the 12-month period pre- and postoperatively. In this way, the effect of the PPV on the average percentage of patients per month who had an uveitic exacerbation, and the average number of medications required per patient per month to control the disease could be assessed. Immunosuppressive agents were classed as topical, orbital, or systemic, and the uveitic episodes were recorded anatomically as anterior uveitis, intermediate uveitis, or posterior.

A standard three-port PPV was performed on all of the eyes. Any further operative manoeuvres were performed as clinically indicated. Lensectomy, IOL implantation, epiretinal membrane peel, retinectomy, fluid/silicone oil or gas exchange, scleral buckling, and choroidal biopsy were performed according to the surgical indication. All operations were performed or directly supervised by one of the four surgeons. Preoperatively, patients were commenced on systemic prednisolone $40 \mathrm{mg}$ /day for 2 weeks if not already on systemic steroids. This was tailed off over 4-8 weeks postoperatively. Where there was a contraindication to systemic steroids, topical and/or orbital steroids were increased in the immediate pre- and postoperative periods.

The eyes were examined preoperatively and at regular intervals for 12 months postoperatively. The clinical information was retrieved from the patient case notes and had been recorded by multiple observers over the time period chosen for the study. Comparisons of categorical data between subgroups of eyes were made using the two-tailed Fisher's exact test and between numerical variables using the Mann-Whitney $U$ test.

\section{Results}

\section{Peroperative}

No major peroperative complications were recorded. A posterior vitreous detachment (PVD) was present in $23 / 41(56 \%)$ of eyes at the time of operation. A PVD was induced peroperatively in the remainder by suction at the edge of the optic disc in seven (17\%) eyes and by surgical delamination in $11(27 \%)$ eyes.

\section{Visual acuity}

Overall, 25/41 (61\%) of the eyes gained more than 2 Snellen lines by 6 months $(P<0.001)$. This improvement was maintained at 12 months (Figure 1). All subgroups, whether analysed according to the indication for surgery or uveitic syndrome, had statistically significant numbers of eyes gaining more than 2 Snellen lines over this time period and no eyes lost more than 2 Snellen lines (Table 1). The number of eyes where the level of vision was $6 / 24$ or less was reduced from 38 to $14(P<0.001)$, and the number of eyes achieving a level of vision of 6/ 12 or greater increased from 0 to $23(P<0.001)$. The visual 
results for the various subgroups did not significantly differ from the pooled results at any time point after the PPV (Table 1).

\section{Cystoid macular oedema}

Cystoid macular oedema (CMO) was significantly reduced after PPV from $18(44 \%)$ of the eyes preoperatively compared to $8(19 \%)$ at 12 months postoperatively $(P<0.05)$ (Figure 1$)$. There was a postoperative reduction of CMO observed in all the uveitic subgroups (Table 2). Resolution of CMO was not associated with a significantly greater visual improvement, with a gain of more than 2 Snellen lines observed in eight of 11 eyes where $\mathrm{CMO}$ resolved and four of seven eyes where it persisted $(P>0.1)$. In the eyes with a pre-existing PVD, CMO was present in 9/23 (39\%) preoperatively and $5 / 23(22 \%)$ at 12 months. Where the

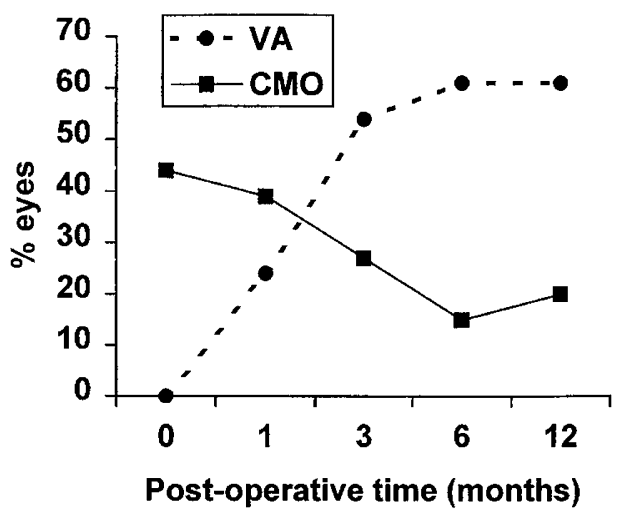

Figure 1 Percentage of eyes gaining more than 2 Snellen lines and percentages of eyes with clinically detectable $\mathrm{CMO}$ against time after PPV for chronic EPU. vitreous was attached, CMO was present in 9/18 (50\%) eyes preoperatively and $3 / 18(17 \%)$ at 12 months. The presence or absence of a PVD did not affect the incidence of CMO either pre- or postoperatively $(P>0.1)$.

\section{Visually significant floaters}

In 18 eyes, the clinical indication for a PPV was visually significant vitreous debris in the absence of vitreous haemorrhage. Lens/capsular opacities were present in seven of these eyes, four with cataracts requiring lensectomy with ciliary sulcus fixated IOL implantation and three with significant posterior capsular opacification requiring surgical capsulotomy combined with an IOL exchange in one case.

The median grade of preoperative vitreous haze was 3 for the 18 eyes with visually symptomatic floaters. After removal/absorption of postoperative intracavity haemorrhage and debris, all eyes improved to grade 0 by 1 month. Symptoms of visually disturbing floaters were relieved in all 18 eyes where this was the primary indication for the PPV $(P<0.001)$. Apart from two transient recurrences of intermediate uveitis, the vitreous cavity remained clear over 12 months follow-up in all cases.

A gain of more than 2 Snellen lines was observed in 11 $(61 \%)$ of these eyes at 12 months $(P<0.001)$. The remaining seven eyes retained their preoperative visual acuity. The incidence of CMO did not significantly reduce over the study period, from nine eyes preoperatively to six eyes at 12 months $(P>0.1)$.

Of the 11 eyes with visually significant vitreous floaters in the absence of lens opacity seven (64\%) gained more than 2 Snellen lines. This improvement was attained in four of the seven (57\%) eyes requiring surgery for concomitant lens/capsular opacities. Removal of lens/

Table 1 Number of eyes gaining more than 2 Snellen lines after PPV for chronic EPU according to subgroup

\begin{tabular}{|c|c|c|c|c|}
\hline & \multicolumn{4}{|c|}{ Number of eyes (\%) gaining $>2$ lines of acuity after PPV } \\
\hline & 1 month & 3 months & 6 months & 12 months \\
\hline \multicolumn{5}{|l|}{ Uveitic group } \\
\hline Intermediate (19) & $3(16)$ & $8(42)^{*}$ & $12(63)^{*}$ & $12(63)^{*}$ \\
\hline Posterior (9) & $5(56)^{*}$ & $8(89)^{*}$ & $7(78)^{*}$ & $8(89)^{*}$ \\
\hline Panuveitis (13) & $2(15)$ & $6(46)^{*}$ & $6(46)^{*}$ & $5(38)^{*}$ \\
\hline All eyes (41) & $10(24)^{*}$ & $22(54)^{*}$ & $25(61)^{*}$ & $25(61)^{*}$ \\
\hline \multicolumn{5}{|l|}{ Indication for $P P V$} \\
\hline Floaters (18) & $5(28)^{*}$ & $10(56)^{*}$ & $11(61)^{*}$ & $11(61)^{*}$ \\
\hline Retinal detachment (8) & $0(0)$ & $3(38)$ & $4(50)^{*}$ & $4(50)^{*}$ \\
\hline Epiretinal membrane (9) & $1(11)$ & $3(56)^{*}$ & $6(67)^{*}$ & $6(67)^{*}$ \\
\hline Vitreous haemorrhage (6) & $4(67)^{*}$ & $4(67)^{*}$ & $4(67)^{*}$ & $4(67)^{*}$ \\
\hline
\end{tabular}

*Denotes statistical significance $(P<0.05)$. 
Table 2 Number of eyes with clinical CMO after PPV for chronic EPU according to subgroup

\begin{tabular}{|c|c|c|c|c|c|}
\hline & \multicolumn{5}{|c|}{ Number of eyes (\%) with clinical CMO after PPV } \\
\hline & Preop & 1 month & 3 months & 6 months & 12 months \\
\hline \multicolumn{6}{|l|}{ Uveitic group } \\
\hline Intermediate (19) & $9(22)$ & $9(22)$ & $6(15)$ & $3(7)$ & $4(10)$ \\
\hline Posterior (9) & $3(7)$ & $2(5)$ & $1(2)$ & $0(0)$ & $0(0)$ \\
\hline Panuveitis (13) & $6(15)$ & $5(12)$ & $4(10)$ & $3(7)$ & $4(10)$ \\
\hline All eyes (41) & $18(44)$ & $16(39)$ & $11(27)^{*}$ & $6(15)^{*}$ & $8(20)^{*}$ \\
\hline \multicolumn{6}{|l|}{ Indication for $P P V$} \\
\hline Floaters (18) & $9(22)$ & $9(22)$ & $6(14)$ & $4(10)$ & $6(15)$ \\
\hline Retinal detachment (8) & $4(10)$ & $4(10)$ & $3(7)$ & $2(5)$ & $2(5)$ \\
\hline Epiretinal membrane (9) & $5(12)$ & $3(7)$ & $2(5)$ & $0(0)^{*}$ & $0(0)^{*}$ \\
\hline Vitreous haemorrhage (6) & $0(0)$ & $0(0)$ & $0(0)$ & $0(0)$ & $0(0)$ \\
\hline
\end{tabular}

*Denotes statistical significant change from preoperative value $(P<0.05)$.

capsular opacities did not significantly increase the chance of gaining more than 2 Snellen lines $(P>0.1)$.

\section{Retinal detachment}

A retinal detachment (RD) was present in 12 eyes, seven presented with tractional RD (TRD), four with combined rhegmatogenous/traction RD (R/TRD), and one with a rhegmatogenous RD (RRD). Four eyes had panuveitis and five had posterior uveitis owing to retinal vasculitis. Intermediate uveitis was present in the remaining three eyes, including the single case of RRD. Overall, six (50\%) of these eyes gained more than 2 Snellen lines, the remainder retained their preoperative visual acuity at 12 months, and this improvement was statistically significant $(P<0.01)$.

The indication for surgery in the eyes with TRD was sudden loss of vision owing to vitreous haemorrhage in four cases and a gradual loss of vision owing to macular detachment in three cases. All seven eyes underwent a PPV with delamination and laser. A further operation with an encircling scleral buckle and silicone oil internal tamponade was required in one eye for persistent retinal traction; the oil was successfully removed at 6 months, with a good anatomical result and a visual acuity of 6/18. Six eyes gained more than 2 Snellen lines at 1 year, with three attaining a final visual acuity of $6 / 12$ or better. The only eye that did not improve was known to have a macular scar prior to surgery.

All four eyes with combined R/TRD underwent PPV and surgical delamination. An encircling buckle was employed in three eyes; two of these had silicone oil and one C3F8 gas internal tamponade. Retinectomy with silicone oil tamponade was performed in one eye. A further delamination and silicone oil top-up procedure was performed in one eye for residual RD. The retina was successfully attached in all cases at 12 months, although the silicone oil was not removed from any eye and one eye became hypotonous (IOP $<5 \mathrm{mmHg}$ ).

None of the eyes had either a preoperative visual acuity of greater than counting fingers $(\mathrm{CF})$ or a gain of more than 2 Snellen lines postoperatively. The single eye with an RRD had macular involvement and was treated with a PPV, scleral buckling, and gas internal tamponade. The retina was successfully reattached although the visual acuity did not improve from $C F$.

\section{Epiretinal membrane (ERM)}

Metamorphopsia and reduced visual acuity as a result of symptomatic ERM were present in nine eyes of nine patients. The ERM were presumed to be secondary to the ocular inflammatory process; five associated with idiopathic panuveitis, two with pars planitis, and two with posterior uveitis. A PPV and membrane peeling procedure was performed in all cases, one eye required a further membrane peel at 2 months postoperatively. A gain of more than 2 lines of acuity at 1 year was noted in six $(67 \%)$ of the eyes $(P<0.005)$, metamorphopsia was subjectively reduced in all eyes, and none of the eyes deteriorated. $\mathrm{CMO}$ was present preoperatively in five eyes and was undetectable clinically in any eye at 1 year $(P<0.05)$.

\section{Postoperative complications}

Early complications (at less than 3 months) included one eye that required a second membrane peel and one a second delamination procedure. One eye developed an RD from an entry site break, which was successfully treated with cryopexy, scleral buckling, and a fluid/gas exchange. One eye that had pre-existent glaucoma 
managed medically required a trabeculectomy augmented with mitomicin-C for a persistent intraocular pressure rise.

Late complications occurring between 3 and 12 months included one eye, which required a cataract extraction and lens implant procedure and one eye, originally treated for a combined TRD/RRD, which became hypotonous. Three eyes developed pigmented macular scars on the postoperative resolution of gross, chronic CMO. Two of these eyes had tractional fibrovascular membranes and one had an ERM removed. These three eyes had a postoperative visual acuity of CF. Late recurrence of CMO at 9-12 months occurred in two eyes, which had final visual acuities of $6 / 60$ and $6 / 36$.

\section{Immunosuppressive agents}

There was no statistically significant difference in the percentage of eyes per month that received systemic or orbital floor immunosuppressive agents over the preand postoperative 12-month periods. There was a statistically significant increase in the use of topical medication over the same period. The percentage of eyes per month not requiring any immunosuppression did not statistically differ over the two time periods (Table 3).

\section{Disease activity}

There was a significant decrease in episodes of intermediate uveitis, posterior uveitis or panuveitis in the postoperative 12-month period compared with the same period preoperatively. The percentage of eyes per month with anterior uveitis increased, although this did not reach statistical significance (Table 3 ). There was a

Table 3 Comparison of immunosuppressive agent use and disease activity in the pre- and postoperative 12-month periods

\begin{tabular}{lccc}
\hline Immunosuppression & $\begin{array}{c}\text { Preoperative } \\
12 \text { months } \\
(\% \text { per month })\end{array}$ & $\begin{array}{c}\text { Postoperative } \\
12 \text { months } \\
(\% \text { per month })\end{array}$ & P value \\
\hline Immunosuppressive agent use & & & \\
None & 30 & 30 & 0.4065 \\
Systemic & 42 & 41 & 0.5702 \\
Orbital & 1 & 0.6 & 0.2975 \\
Topical & 31 & 46 & $0.0349^{*}$
\end{tabular}

Uveitic exacerbations

Uveitic syndrome

$\begin{array}{llcl}\text { Anterior } & 5 & 13 & 0.1437 \\ \text { Intermediate } & 9 & 1 & 0.0001^{*} \\ \text { Posterior } & 9 & 1 & 0.0003^{*} \\ \text { Panuveitis } & 7 & 0.4 & 0.0003^{*}\end{array}$

*Statistically significant change in pre- and postoperative value $(P<0.05)$. significant increase in the percentage of eyes per month that did not suffer any episodes of uveitis, from 70 to $84 \%$ $(P=0.0012)$.

\section{Discussion}

In our study, PPV combined with appropriate adjunctive surgical manoeuvres was safe and effective in the management of the complications of chronic EPU. A gain of more than 2 Snellen lines was observed in $61 \%$ of eyes, with $75 \%$ attaining a final visual acuity of $6 / 24$ or better and $56 \%$ attaining $6 / 12$ or better. This compares well with other smaller series of the effects of PPV on the course of chronic uveitis, where an improvement of more than 2 Snellen lines was observed in $48-83 \%$ of eyes with $48-75 \%$ achieving $6 / 24$ or better and $32-50 \%$ achieving $6 / 12$ or better. $6,8,15$

Visual improvement from the PPV is produced by a number of factors. These include the physical clearance of vitreous inflammatory debris, the reduction of $\mathrm{CMO}$, and the anatomical correction of sight-threatening retinal abnormalities. Clearance of vitreous debris was a major visual factor for the subgroups where PPV was performed for visually significant vitreous floaters and vitreous haemorrhage. Anatomical factors were likely to be more important in the groups where the PPV was performed for RD and ERM. The likelihood of gaining more than 2 Snellen lines did not significantly differ for any of the subgroups. The RD group took longer to achieve a significant improvement of acuity, and the overall acuity tended to be worse with $50 \%$ achieving a final Snellen acuity of 6/ 24 or better and $33 \%$ attaining $6 / 12$ or better. The group of four eyes with combined TRD/RRD had the worst results; none of these eyes gained more than 2 lines of visual acuity, all but one eye required longterm silicone oil tamponade for residual peripheral RD.

Clinically detectable CMO decreased by $56 \%$ in a fashion similar to other studies where a $40-50 \%$ reduction was seen. ${ }^{6,8,9}$ Clinical assessment of CMO in a retrospective series is subject to the inaccuracies of the objectivity of the examination and the difficulties of fundal viewing through vitreous debris. Nevertheless, the authors believe that the findings of this study represent a true therapeutic effect of PPV and deserve further investigation. It should be noted that prospective studies retain the inherent difficulties of assessment of $\mathrm{CMO}$ behind opacified media. Late postoperative recurrences of $\mathrm{CMO}$ are recognised and occurred in two eyes in our study. ${ }^{17}$ The clinical resolution of $\mathrm{CMO}$ was not statistically associated with visual improvement, suggesting that other factors such as the clearance of vitreous debris are responsible for the visual 
improvement seen in this series. It may be that earlier surgical intervention is required to influence the visual outcome associated with CMO resolution. Improvement of CMO was not proven angiographically and in some eyes may have persisted despite the clinical fundal appearance. Although clinical resolution implies at least a reduction in oedema, this may not have been sufficient to allow a visual improvement. Furthermore, in some cases, irreversible macular damage may have already occurred as a result of the chronic $\mathrm{CMO}$; this is illustrated by the three eyes that developed pigmented macular scars on resolution of the CMO.

ERM formation is a common cause of visual loss for patients with EPU. Malinowski et al ${ }^{18}$ found that $30 \%$ of patients with pars planitis developed ERM of which $6.5 \%$ were classified as severe. Dev et al ${ }^{19}$ performed PPV with membrane peeling on seven eyes with pars planitis and ERM. A visual improvement of more than 2 Snellen lines was attained in $71 \%$ of the eyes. ${ }^{19}$ Our study has similar findings, with six $(67 \%)$ of nine eyes achieving the same visual improvement.

An improvement in the course of uveitis after PPV has been noted in other studies, and this improvement is associated with a reduction in the use of medications to control the disease process. ${ }^{9-13}$ In our study, the number and classes of medications did not change in the postoperative period. This difference may be explained by the increased use of immunosuppression in the immediate postoperative period. With the use of perioperative systemic steroids and careful observation of the eyes in the immediate postoperative period, the eyes in this series did not suffer from uncontrolled uveitic episodes postoperatively. The episodes of anterior uveitis seen postoperatively (Table 3) were generally mild and easily controlled. It is possible that following the reduction in postoperative medications, a significant overall reduction in immunosuppression would be seen in the second postoperative year.

There was a significant reduction in uveitic exacerbations in the posterior segment postoperatively. There is evidence that the population of autoreactive lymphocytes lodged in the vitreous of eyes with EPU is unable to exit the eye and does not appear to undergo apoptosis. The reduction of inflammatory relapses and CMO after PPV may be through the removal of these cells, abolishing the abnormal Th1/Th2 balance of lymphocytes within the uveitic eye which is proposed to mediate uveitic exacerbation and remission. ${ }^{10}$ The continued episodes of anterior uveitis postoperatively may be caused by the stimulus of surgical trauma, but could potentially also be because of abnormalities in immune regulation in anterior uveitis that are not affected by removal of vitreous cells.
Another potential beneficial effect of PPV in uveitis is the replacement of the vitreous with aqueous humour which itself has anti-inflammatory effects. There is a decreased level of cortisol binding globulin (TBG) in the aqueous humour compared to the plasma, increasing the free concentration of cortisol to enhance its immunosuppressive effects. ${ }^{20}$ Additionally, aqueous humour constitutively expresses the anti-inflammatory cytokines TGF- $\beta$, vasoactive intestinal peptide (VIP) and $\alpha$-melanocyte stimulating hormone $(\alpha-\mathrm{MSH}){ }^{21-23}$

Aqueous humour also inhibits complement fixation through an unknown mechanism and promotes apoptosis to allow the removal of inflammatory cells after an inflammatory episode. ${ }^{24,25}$

The results of this study add to the evidence that PPV has a beneficial effect on the clinical course of EPU. We did not evaluate subjective changes in vision following surgery. Our clinical impression was that this often improved even when the patient's measured visual acuity was not greatly increased.

\section{References}

1 Forrester JV. Uveitis: pathogenesis. Lancet 1992; 388: 1498-1501.

2 Dick AD. Experimental approaches to specific immunotherapies in autoimmune disease: future treatment of endogenous posterior uveitis?. Br J Ophthalmol 1995; 79: 81-88.

3 Chan CC, Matteson DM, Li Q, Whitcup SM, Nussenblatt RB. Apoptosis in patients with posterior uveitis. Arch Ophthalmol 1997; 115: 1559-1567.

4 Hultsch E. Modulation of lymphocyte stimulation by the vitreous body. A new concept in ocular immunology. Dev Ophthalmol 1981; 2: 8-14.

5 Dick AD. The treatment of chronic uveitic macular oedema. Is immunosuppression enough? Br J Ophthalmol 1994; 78: $1-2$.

6 Heiligenhaus A, Bornfeld N, Foerster MH, Wessing A. Long-term results of pars plana vitrectomy in the management of complicated uveitis. Br J Ophthalmol 1994; 78: 549-554.

7 Fox GM, Flynn HW, Davis JL, Culbertson W. Causes of reduced visual acuity on long-term follow-up after cataract extraction in patients with uveitis and juvenile rheumatoid arthritis. Am J Ophthalmol 1992; 114: 708-714.

8 Mieler WF, Will BR, Lewis H, Aaberg TM. Vitrectomy in the management of peripheral uveitis. Ophthalmology 1988; 95: 859-864.

9 Kloti R. Pars plana vitrectomy in chronic uveitis. Klin Monatsbl Augenheilkd 1988; 192: 425-429.

10 Franks WA, Limb GA, Stanford MR, Ogilvie J, Wolstencroft $\mathrm{RA}$, Chignell $\mathrm{AH}$ et al. Cytokines in human intraocular inflammation. Curr Eye Res 1992; 11(S): 187-191.

11 Bovey EH, Gonvers M, Herbort CP. Pars plana vitrectomy in uveitis. Klin Monatsbl Augenheilkd 1992; 200: 464-467.

12 Messerli J, Korner F, Ruggli J. Chronic uveitis: course after vitrectomy. Klin Monatsbl Augenheilkd 1992; 200: 378-381. 
13 Libert J. Vitrectomy in uveitis. Bull Soc Belge Ophthalmol 1989; 230: 143-153.

14 Verbraeken H. Diagnostic vitrectomy and chronic uveitis. Graefes Arch Clin Exp Ophthalmol 1996; 234(S): 2-7.

15 Verbraeken $\mathrm{H}$. Therapeutic pars plana vitrectomy for chronic uveitis: a retrospective study of the long-term results. Graefes Arch Clin Exp Ophthalmol 1996; 234: 288-293.

16 Nussenblatt RB, Palestine AG, Chan CC, Roberge F. Standardisation of vitreal inflammatory activity in intermediate and posterior uveitis. Ophthalmology 1985; 92: 467-471.

17 Eckardt C, Bacskulin A. Vitrectomy in intermediate uveitis. Dev Ophthalmol 1992; 23: 232-238.

18 Malinowski SM, Pulido JS, Folk JC. Long-term visual outcome and complications associated with pars planitis. Ophthalmology 1993; 100: 818-825.

19 Dev S, Mieler WF, Pulido JS, Mittra RA. Visual outcomes after pars plana vitrectomy for epiretinal membranes associated with pars planitis. Ophthalmology 1999; 106: 1086-1090.

20 Knisely TL, Hosoi J, Nazareno R, Granstein RD. The presence of biologically significant concentrations of glucocorticoids but little or no cortisol binding globulin within aqueous humor: relevance to immune privilege in the anterior chamber of the eye. Invest Ophthalmol Vis Sci 1994; 35: 3711-3723.

21 Cousins SW, McCabe MM, Danielpour D, Streilein JW. Identification of transforming growth factor-beta as an immunosuppressive factor in aqueous humor. Invest Ophthalmol Vis Sci 1991; 32: 2201-2211.

22 Taylor AW, Streilein JW, Cousins SW. Identification of alphamelanocyte stimulating hormone as a potential immunosuppressive factor in aqueous humor. Curr Eye Res 1992; 11: 1199-1206.

23 Taylor AW, Streilein JW, Cousins SW. Immunoreactive vasoactive intestinal peptide contributes to the immunosuppressive activity of normal aqueous humor. $J$ Immunol 1994; 153: 1080-1086.

24 Mondino BJ, Sumner H. Complement inhibitors in normal cornea and aqueous humor. Invest Ophthalmol Vis Sci 1984; 25: 483-486.

25 D'Orazio TJ, DeMarco BM, Mayhew ES, Niederkorn JY. Effect of aqueous humour on apoptosis of inflammatory cell types. Invest Ophthal Vis Sci 1999; 40: $1418-1425$ 